\title{
A New Method for Breath Capture Inside a Space Suit Helmet
}

\author{
Tom Filburn, Craig Dolder, Brett Tufano \\ University of Hartford, West Hartford CT \\ Heather L. Paul \\ NASA Johnson Space Center, Houston TX
}

Copyright (C) 2007 SAE International

\begin{abstract}
This project investigates methods to capture an astronaut's exhaled carbon dioxide $\left(\mathrm{CO}_{2}\right)$ before it becomes diluted with the high volumetric oxygen flow present within a space suit. Typical expired breath contains $\mathrm{CO}_{2}$ partial pressures $\left(\mathrm{pCO}_{2}\right)$ in the range of 20-35 $\mathrm{mm} \mathrm{Hg}$. This research investigates methods to capture the concentrated $\mathrm{CO}_{2}$ gas stream prior to its dilution with the low $\mathrm{pCO}_{2}$ ventilation flow. Specifically this research is looking at potential designs for a collection cup for use inside the space suit helmet.
\end{abstract}

The collection cup concept is not the same as a breathing mask typical of that worn by firefighters and pilots. It is well known that most members of the astronaut corps view a mask as a serious deficiency in any space suit helmet design. Instead, the collection cup is a non-contact device that will be designed using a detailed Computational Fluid Dynamic (CFD) analysis of the ventilation flow environment within the helmet. The CFD code, Fluent, provides modeling of the various gas species $\left(\mathrm{CO}_{2}\right.$, water vapor, and oxygen $\left.\left(\mathrm{O}_{2}\right)\right)$ as they pass through a helmet. This same model will be used to numerically evaluate several different collection cup designs for this same $\mathrm{CO}_{2}$ segregation effort. A new test rig will be built to test the results of the CFD analyses and validate the collection cup designs. This paper outlines the initial results and future plans of this work.

\section{INTRODUCTION}

NASA has produced a detailed plan for human return to the moon. This plan includes two new launch vehicles (Ares I and Ares V) which will provide both human-rated and heavy lift launch capabilities respectively. While planning for these new missions, NASA engineers have recognized that new hardware will be required to achieve the mission goals, and minimize weight and volume of all systems.

Among the new hardware requirements will be the need for a space suit to allow explorers the opportunity to complete missions in which extravehicular activities
(EVAs) will be conducted in microgravity, and on the surfaces of the Moon and Mars. Based on the need to perform these future exploration missions in the lunar gravity environment (1/6 Earth gravity), minimizing onback life support system weight will be critical to achieving NASA's lunar exploration plan. These weight goals become even more critical if a space suit will be used to traverse the Mars surface, as Mars gravity is $1 / 3$ of Earth gravity.

The existing shuttle Extravehicular Mobility Unit (EMU) has been designed for, built, and is still operated within a microgravity environment. With a mass of approximately $125 \mathrm{~kg}$, this suit weighs nearly double that worn by astronauts in the original Apollo program during their lunar surface sojourns. Therefore, NASA recognizes that numerous weight reduction activities will be required to produce a new space suit for lunar surface exploration. While the Apollo space suits were adequate for the lunar missions, the Constellation program envisions longer lunar stay times and a greater number of EVAs. Therefore, simply duplicating the Apollo suit will not be sufficient to accomplish the planned lunar surface forays for Constellation.

This project investigates a new method to reduce the weight associated with the $\mathrm{CO}_{2}$ capture and moisture control system. Specifically, a device will be designed for capturing the exhaled breath of a suited astronaut, as this exhaled breath contains a high concentration of water vapor and carbon dioxide.

\section{DISCUSSION}

Any contaminant capture system or purge ventilation system will operate most efficiently when ventilation contaminants are at high concentrations. Therefore, if a system were in place to capture the exhaled breath prior to dilution with the ventilation flow, overall ventilation subsystem, size, weight and power requirements could be reduced. For instance, the six absolute cubic feet per minute (acfm) ventilation flow rate of the EMU was selected based on studies that evaluated $\mathrm{CO}_{2}$ washout from the oral/nasal region. This six acfm flow rate also 
prevented moisture from condensing on the faceplate of the helmet. However, a breathing cup, situated near the mouth could provide a sink for the expired $\mathrm{CO}_{2}$ and water vapor. This sink would collect these contaminants more efficiently than relying on a high volumetric washout flow. Therefore, the effect would be a decrease in the required ventilation flow. A lower flow rate would allow for a smaller fan, as well as a reduction in the battery size (or an increase in EVA duration).

Within the present space suit helmet, the $\mathrm{CO}_{2}$ concentration in the oral/nasal region of the crewmember is controlled by continuously flowing six acfm oxygen over the crewmember's head and face. This flow rate was determined via analysis to be sufficient to keep the inspired $\mathrm{CO}_{2}$ concentration below $7.6 \mathrm{~mm} \mathrm{Hg}$ for most metabolic rates. At the extremely high work regime, this flow rate is sufficient to keep the inspired $\mathrm{CO}_{2}$ below $15 \mathrm{~mm} \mathrm{Hg}$. As seen in Figure 1 , both of these limits $(7.6 \mathrm{~mm} \mathrm{Hg}(0.01 \mathrm{~atm})$ and $15 \mathrm{~mm}$ $\mathrm{Hg}(0.02 \mathrm{~atm}))$ are sufficient to prevent any impact to the astronaut's health or performance.

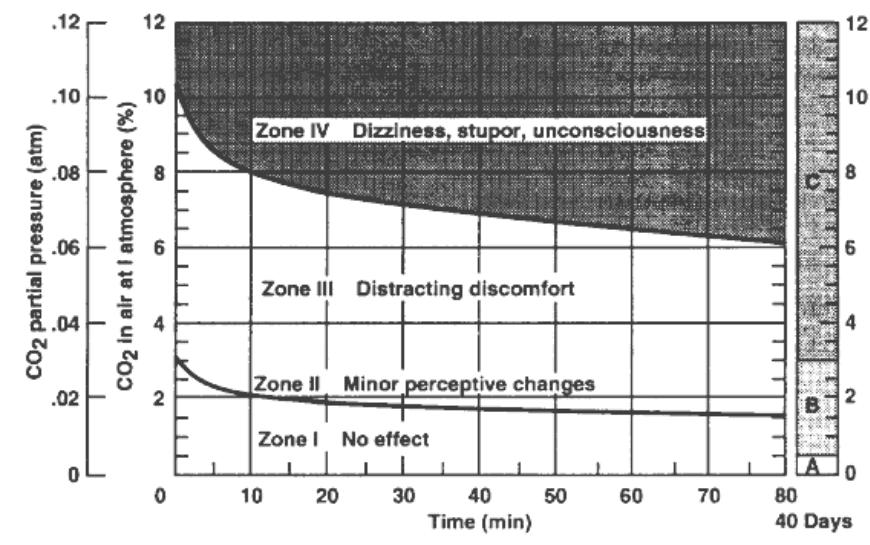

Figure 1. Short Term and Long Term Effects of $\mathrm{CO}_{2}$ concentration $^{1}$

While the $\mathrm{CO}_{2}$ concentration within the helmet can be controlled by sufficient ventilation flow, the result is dilution of $\mathrm{CO}_{2}$ into the suit volume, and therefore the need for a $\mathrm{CO}_{2}$ control system capable of removing the low partial pressure $\mathrm{CO}_{2}$ from the ventilation stream. Figure 2 shows that the concentration of $\mathrm{CO}_{2}$ in an average expired breath is two to four times the 7.6 and $15 \mathrm{~mm} \mathrm{Hg}$ limits set for a crewmember's inspired breathing gas. While these high concentrations will negatively impact an astronaut's health if inspired, capturing these same high concentrations prior to dilution will provide a higher driving force for $\mathrm{CO}_{2}$ control. Therefore $\mathrm{CO}_{2}$ adsorbents will achieve higher capture efficiencies when operated with the increased $\mathrm{CO}_{2}$ concentrations found in an astronaut's exhalant.

The proposed breathing cup will remove this contaminant from the oral/nasal region reducing the need for ventilation flow to "sweep" it away. In addition to reducing ventilation flow requirements, thereby reducing fan size and weight, the PLSS weight can also be reduced via the decreased fan power requirement. The lower flow fan will have a lower power draw, allowing for a smaller battery (or alternative power supply e.g. fuel cell) to be used.

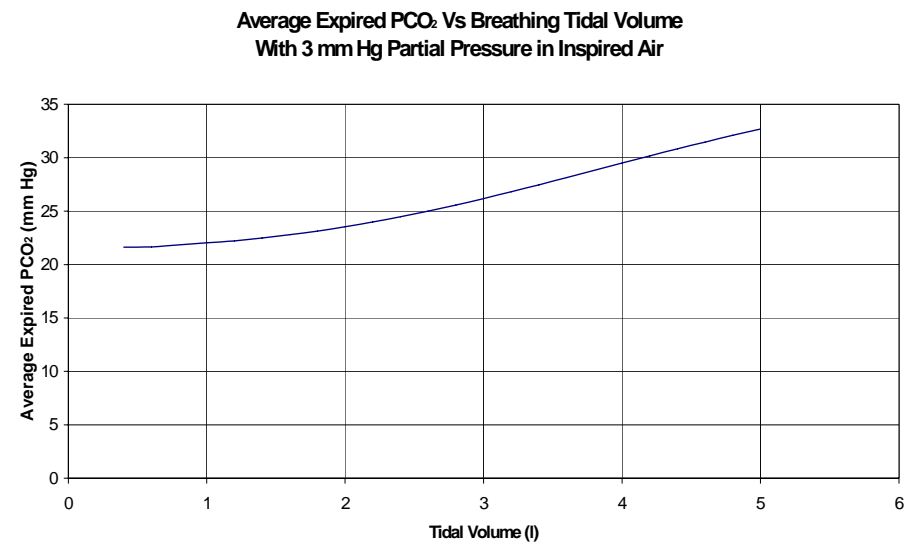

Figure 2. Average $\mathrm{CO}_{2}$ Concentration in exhalant

The overall task is to produce a prototype breath capture device for the helmet. This program has been devolved into five steps, of which four have been initiated. In step one, a CFD model of the bubble helmet presently used in the EMU has been developed. A CFD model of a hemispherical helmet proposed for future space suit designs will be created during the summer of 2007. Step two of the program assessed the compatibility of a breathing cup with various $\mathrm{CO}_{2}$ capture systems (Trade Study). Step three of the program uses the CFD analysis to design a breath capture device. Step four will corroborate the CFD model by physical testing of the prototypes that will be manufactured. Step five will be a final assessment of the feasibility of this breath capture device and delivery of at least one prototype article to the NASA Johnson Space Center.

\section{STEP 1 CFD Model Development}

In order to provide a design tool for assessing the ability of a breathing cup to operate within a space suit helmet, a CFD model of the ventilation and respiration flows within the helmet was developed. This program used the CFD software Fluent. Fluent is divided into two subparts. GAMBIT is the software used to create and mesh the physical geometry, and also set boundary conditions. Fluent is the code used to solve the continuity, momentum and energy equations for either laminar or turbulent flow. The three dimensional (3-D) CFD model was constructed in GAMBIT from measurements of the bubble helmet. The first model was run in Fluent with a steady state model consisting of a generic gas mixture. The fresh oxygen inlet at the back of the helmet and the mouth were modeled as having a constant velocity. The outlet into the general suit circulation was modeled as a constant pressure at one atmosphere. Future model enhancements will include setting the operating pressure equal to the $29.5 \mathrm{kPa}$ 
expected for this future exploration suit. For the next step of complexity, a sinusoidal velocity profile will be applied to the mouth in order to simulate breathing. The solver will be changed from steady state to timedependent in order to process the unsteady breathing model. Figure 3 shows a view of the bubble helmet geometry created in GAMBIT, and the elliptical tube that was used as the breathing cup. The CFD modeling effort has concentrated on the bubble helmet configuration. It is expected, that a CFD model of the hemispherical helmet will be completed and analyzed during the summer of 2007.

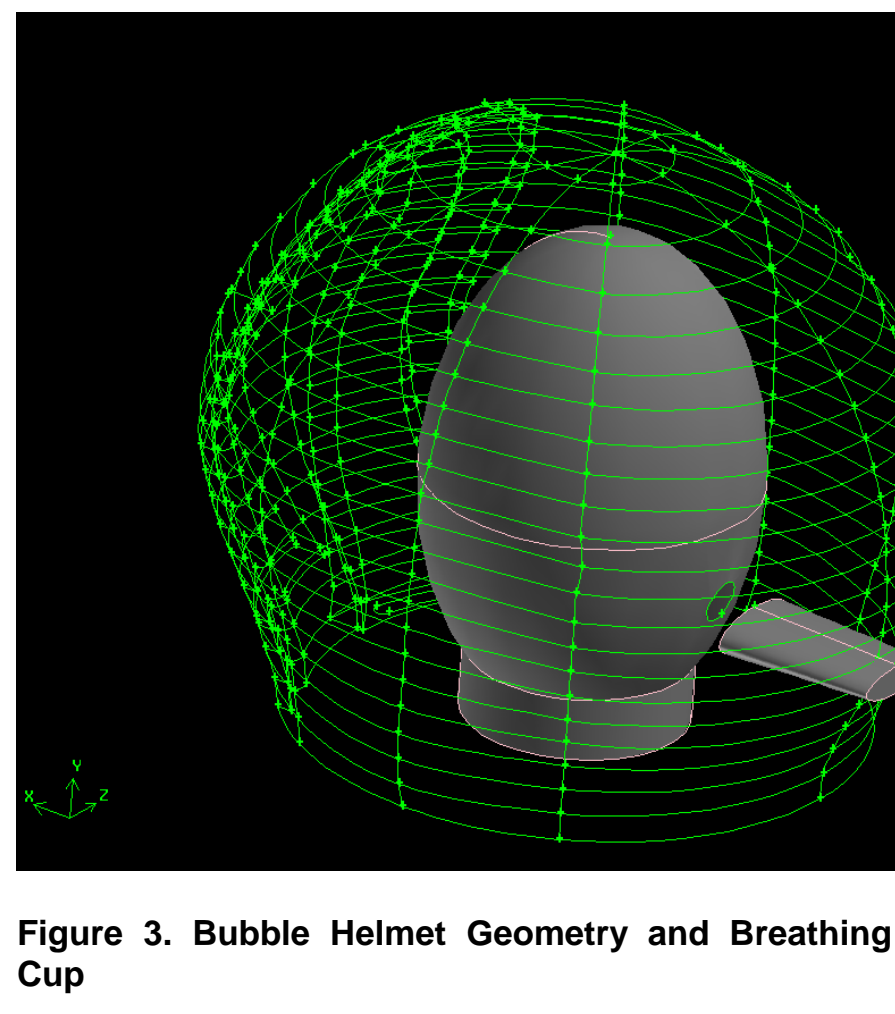

After confirming that the model could represent a simplified astronaut's head, an initial breathing cup model was made. This model converged without using the sinusoidal breathing model but simply assumed a continuous flow out of the mouth. The cup outlet was approximated by an ellipse set at a constant pressure one Pascal $(1 \mathrm{~Pa})$ below that of the general suit circulation outlet.

\section{STEP $2 \mathrm{CO}_{2}$ Capture Trade Study}

A variety of $\mathrm{CO}_{2}$ removal technologies that could be coupled into a PLSS with the prototype breathing cup system were investigated. Table 1 shows the technologies considered in this trade study and various sub-system attributes.

Seven different $\mathrm{CO}_{2}$ removal technologies to combine with the breath capture system were evaluated. These seven systems included a four-bed molecular sieve system, metal oxide sorbent sheets, granular solid amine sorbents, membranes, electrochemical systems, steam desorbed solid amines (Solid Amine Water
Desoprtion, SAWD) and cryogenic freeze-out. All seven of the systems offered a low ventilation flow pressure drop, and therefore would have a low fan power requirement. Those systems with a low Technology Readiness Level (TRL) were discarded due to the complexity of developing two technologies in parallel (breathing cup and $\mathrm{CO}_{2}$ capture system). Of the three remaining $\mathrm{CO}_{2}$ removal systems with a high TRL, the solid amine system offered the best combination of low weight, low volume, and low regeneration energy.

The results of this trade study indicated that a two-bed solid amine pressure swing absorption system would be ading $\mathrm{CO}_{2}$ capture system to include with a ng cup. Discussions with various suit design ers indicated that even lower on-back weights be achieved without any $\mathrm{CO}_{2}$ capture system. rentuality (no $\mathrm{CO}_{2}$ capture system) can only be red if the breath capture system has extremely pture efficiency. This system design, eliminating 2 capture system, would operate with the breath cup automatically venting the high $\mathrm{CO}_{2}$ tration gas directly overboard. Once designs and cies have been determined for the breathing cup $\mathrm{t}$, a system sizing trade study will determine what acts are to the overall PLSS weight, volume, and when comparing venting the exhalant overboard recirculating the captured exhalant through a ntrol system.

\begin{tabular}{|l|l|l|l|l|l|}
\hline \multicolumn{1}{|l|}{ ogy } & $\Delta \mathrm{P}$ & $\begin{array}{l}\text { Regen } \\
\text { Energy }\end{array}$ & Weight & Volume & $\mathrm{TRL}$ \\
\hline S & Low & High & Med & Med & 8 \\
\hline Metox & Low & High & High & Low & 8 \\
\hline Solid Amine & Low & Low & Med & low & 8 \\
\hline Membrane & Low & Low & Low & Low & 4 \\
\hline E Chem & Low & $?$ & High & Low & 3 \\
\hline SAWD & Low & Med & Med & Low & 4 \\
\hline Cryo Freeze & low & Low & low & Low & 4 \\
\hline
\end{tabular}

Table $1 \mathrm{CO}_{2}$ Capture Trade Study

\section{STEP 3 Breathing Cup CFD Analysis}

This section details the efforts to create the CFD model using both the Fluent solver and the GAMBIT preprocessor. The initial CFD model was simplified to allow the generation of a converged solution. These simplifications included setting both input flows to pure gas, i.e. the oxygen inlet was defined as pure $\mathrm{O}_{2}$ and the mouth was defined as expelling pure $\mathrm{CO}_{2}$. This model resulted in an efficiency of $61 \%$ with an uncertainty of $16 \%$, based on the mass imbalance of $\mathrm{CO}_{2}$ within the model relative to the $\mathrm{CO}_{2}$ flow within the breathing cup. This result indicated that the initial breathing cup model 
would capture $61 \%$ of the exhaled $\mathrm{CO}_{2}$. The remaining $\mathrm{CO}_{2}$ would mix with the ventilation gas and leave the helmet through the neck ring opening. Even taking the maximum uncertainty, this model is realistic and can be used as a benchmark between different configurations of the breathing cup.

Different mesh densities were tested to maximize the accuracy of results versus the iteration time. The three mesh densities from highest to lowest that were developed resulted in efficiencies of $71.5 \%, 70.9 \%$ and $69.6 \%$ and mass flow uncertainties of $7.5 \%, 18.4 \%$ and $26.7 \%$, respectively, for the same boundary conditions. Since the model with the lowest mesh density converged in significantly less time than the other models, and the percent difference in $\mathrm{CO}_{2}$ capture efficiency between the most and least accurate model was only $2.7 \%$, the current relationships are being run with the lowest density mesh of the three explored.

The first breathing cup design is an ellipse with a horizontal major diameter of 2" and vertical minor diameter of $3 / 4 "$. It is centered $1 / 2$ " below the center of the mouth and shrinks uniformly at an angle of $2^{\circ}$ to the boundary of the helmet where it ends. This is the first iteration, and variations in size, geometry and location for alternate designs will be evaluated. The concept shown in Figure 3 is positioned 1" in front of the head. Figure 4 shows this initial cup design.

\section{Figure 4. Initial Breathing Cup Design - To be added.}

Parametric relationships will be run to look at the pressure of the breathing cup, the breathing cup distance from the mouth and the shape of the breathing cup inlet. Another measurement for efficiency will also need to be developed, as a breathing cup that has $99 \%$ $\mathrm{CO}_{2}$ capture, but also removes a significant percentage of oxygen from the helmet, would be unacceptable. Figure 5 shows one output from the Fluent program. This figure details the $\mathrm{CO}_{2}$ concentration and flow velocities in the region of the breathing cup.

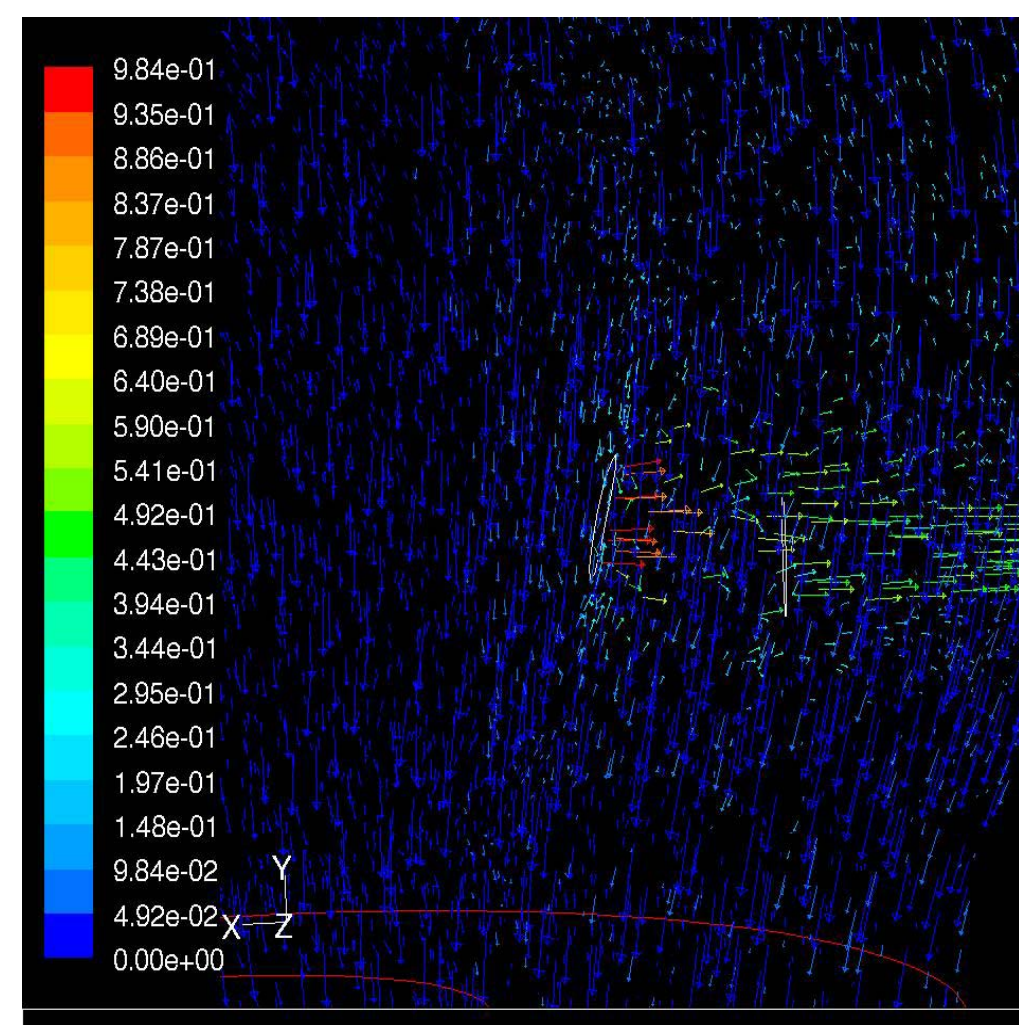

Velocity Vectors Colored By Mass fraction of co2

FLUENT 6.2 (3d, dp,

\section{Figure 5. $\mathrm{CO}_{2}$ Concentration near Breathing Cup}

\section{STEP 4 Prototype Performance Testing}

The overall project plan includes the capability for testing of the breathing cup prototype designs. This testing is intended to validate the CFD model and provide confidence in the $\mathrm{CO}_{2}$ capture performance of the prototype cup designs. Figure 6 shows the test rig schematic that will be used to evaluate the performance of the breathing cup designed from the CFD analyses. While the test rig has not been assembled yet, all of the components necessary for testing the $\mathrm{CO}_{2}$ capture efficiency of the breathing cup have been identified. The plan is to flow the ventilation gas through a bubble or hemispherical helmet at the appropriate flow rate (6 acfm or $4 \mathrm{acfm}$ ). A mannequin head located inside the helmet will be used to simulate a breathing astronaut. This head will be connected to two mass flow controllers which will set nitrogen and $\mathrm{CO}_{2}$ flow rates to simulate variable breathing patterns. This allows the capability of altering the metabolic rate, and therefore the $\mathrm{CO}_{2}$ concentration in the simulated exhalant. The mannequin head will also be capable of repositioning, to simulate an astronaut looking to the right or left. In this fashion, an astronaut altering the head position within the helmet will be evaluated. These changes will be documented, and the effect on the breathing cup efficiency will be documented. 


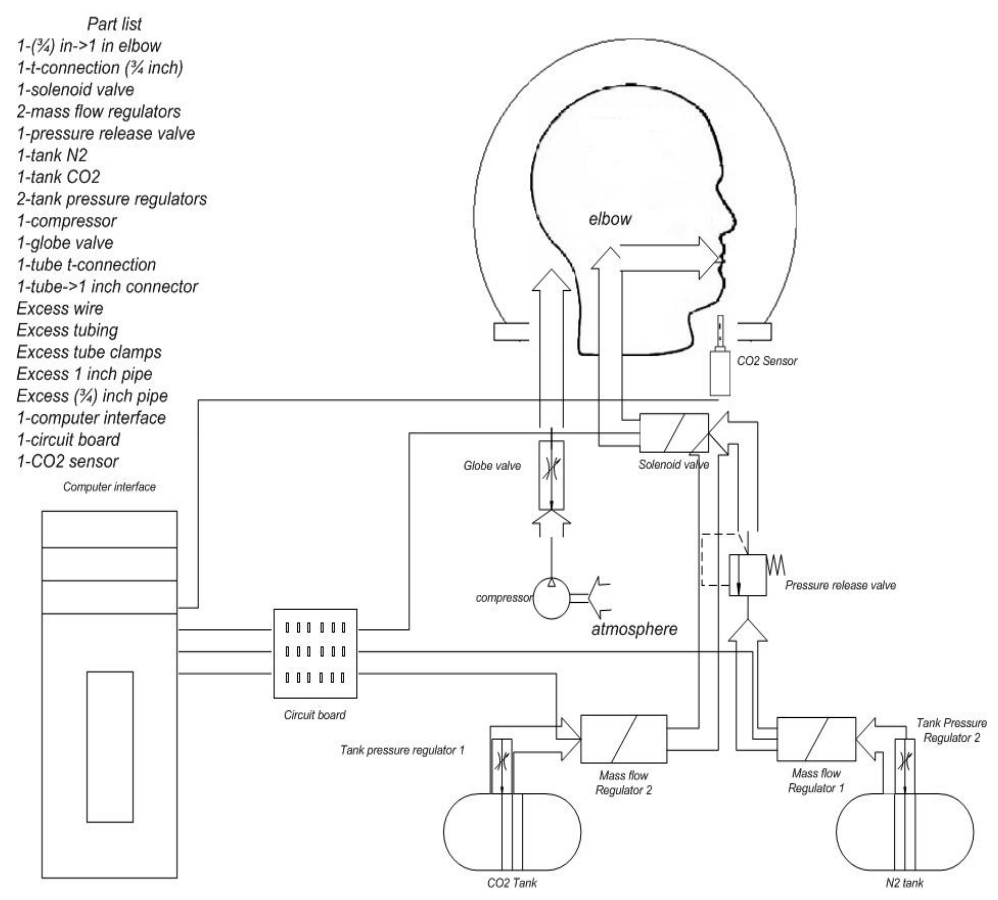

Figure 6. Test Rig Schematic for Evaluating Breathing Cup Prototype Performance

\section{Step 4 Overall Breathing Cup Assessment}

The CFD analyses, prototype design, and prototype performance tests are expected to be iterative processes. That is, the initial results of the CFD analysis will be used to produce a simple StereoLithography (SLA) model for testing in the Environmental Lab at the University of Hartford. The initial results of the test rig will be used to validate the assumptions used in the CFD model. If these results are validated, then further CFD analyses will be performed to optimize the design of the breathing cup. As stated before, optimal performance will be defined as maximizing the capture of $\mathrm{CO}_{2}$. In addition, optimal performance will also need to account for the fraction of $\mathrm{O}_{2}$ gas captured/lost by the breathing cup. If discrepancies appear between the physical and CFD modeling, then revisions to the assumptions will be made to achieve agreement between these two methods of evaluation.

\section{CONCLUSION}

Overall this project seeks to investigate a novel method of breath capture for use inside the helmet of a future space suit. The use of this device (breathing cup) offers the possibility of reduced PLSS complexity and weight. This project has begun a proof-of-concept effort. The detailed CFD analyses of the flow fields within a space suit helmet have begun. The CFD model of a bubble helmet has been completed, and a CFD model of a hemispherical helmet will begin during the summer of 2007. This CFD analysis has been further refined to include a breathing cup that has a slightly lower pressure compared to the neck ring ventilation flow return region. Modeling this flow field to identify the portion of metabolically produced $\mathrm{CO}_{2}$ that is captured by the breathing cup is under way. This CFD analysis will continue to optimize the design of this breathing cup. A laboratory test rig is in the process of being assembled to verify the computer predicted performance of the breathing cup.

\section{ACKNOWLEDGMENTS}

Funding for this effort has been provided by the Crew and Thermal Systems division at the NASA Johnson Space Center.

\section{REFERENCES}

1. NAVSHIPS 0994-003-7010, US Navy Diver Manual

\section{CONTACT}

Tom Filburn

Associate Professor, Mechanical Engineering

University of Hartford

200 Bloomfield Ave

West Hartford CT 06117

filburn@hartford.edu

860-768-4843 